55. Bullinger, M. Quality of life and chronic conditions: theiperspective of children and adolescents in rehabilitation / M. Bullinger, U. Ravens-Sieberer // Prax. Kinderpsychol. Kinderpsychiatr. - 2006. - Vol. 55, № 1. - P. 23-35.

56. Epidemiology of cleft palate in Europe: implications for genetic research / E. Calzolari, F. Bianchi, M. Rubini [et al.] // Cleft Palate Craniofac J. - 2004. - Vol. 41, № 3. - P. 244-246.

57. Flinn W., Long RE., Garattini G., Semb G. A multicenter outcomes assessment patients with unilateral cleft lip and palate. Cleft Palate Craniofac J. 2006 May; 43(3):25-28.

58. Genetic Association Studies of Cleft Lip and/or Palate WithHypodontia Outside the Cleft Region / R. L. Slayton, L. Williams, J.C. Murray [et al.] // The Cleft Palate - Craniofacial Journal. - 2003. - Vol. 40, № 3. - P. 274-279.

59. Harrison, R. Oral health promotion for high-risk children: Case studies from British Columbia/ R. Harrison // J. Can. Dent. Assoc. - 2003. - Vol. 69, № 5. - P. 292-296. 212.Haugejorden, O. Evidence for reversal of the caries decline among Norwegian children / O. Haugejorden, J. M. Birkeland // Int. J. Paediatr. Dent. 2002. - Vol. 12, № 5. - P. 306-315.

60. Hathorn I.S., Atack N.E., Butcher G. et al. Centralization of services: standard setting and outcomes // Cleft Palate Craniofac. J. 2006. - Vol. 43, № 4. -P. 401-405.

61. Hewson, A. R. Dental experience of cleft affected children in the west of Ireland / A. R. Hewson, C. M. McNamara, T. F. Foley // Int. Dent. J. - 2001. -Vol. 51. - P. 7376.

62. Hugentobler M., Dojcinovic I., Richter M. Flap techniques in secondary alveolar between two types of flap. Rev StomatolChirMaxillofac. 2006 Jun; 10 (3): 145-51

63. Lilius GP. Clefts with associated anomalies and syndromes in Finland. Scand J PlastReconstrSurg Hand Surg. 1992; 26:185-196.

64. Mc. Comb. H.K. Coghlan B.A. Primery repair of the unilateral cleft lip nose, completion longitudinal study. CleftPalateCraniofac. J. 1996. jan,33(1) 23-30.

65. Menegotto, B. G. Epidemiology of Oral Clefts in a Large South American Sample / B. G. Menegotto, F. M. Salsano // Cleft Palate Craniofac J. - 1991. - Vol. 28, № 4. - P. 376-377

66. Neumann H. J. Development of lip, jaw, palatal and uvular clefts. // Zahntechnik (Berl). 1990. — 23(6) — P. 271-274.

67. Paul, T. Oral and dental status of children with cleft lip and/or palate / T. Paul, R. S. Brand // The The Cleft Palate-Craniofacial Journal. - 1998. - Vol. 35, № 4. - P. 329332.

68. Recaman M., BonetB., Leitao J., Mesquita. Reconstruction of velopharyngeal speech Cleft Palate: surgical alternative. Cir Pediatr 2006 Apr; 19(2): 106-10.

69. Slifer KL. Pulbrook V., Amari A., Vqna-Ambadar., Beck M., Piszczor R. Socia acceptance and facial behavior clefts. Pediatric Psychology, Department of Behavi. Krieger Institute, 707 N. Broadvway, Baltimo. Cleft Palate Craniofac J 2006 Mar;43(2):22-36.

70. The effectiveness of a 6-year oral health education program for primary schoolchildren / J. Vanobbergen, D. Declerck, S. Mwalili, L. Martens // Community Dent. Oral. Epidemiol. - 2004. - Vol. 32, № 3. - P. 173-182.

71. Tooth loss and dentures: patients' perspectives / Judit A. Jones, Michelle Borner, Avron Spiro III, Nancy R. Kressin // International Dental Journal. - 2003. - Vol. 53. - P. 327-334.

\title{
Докучаева О.А. \\ Аденомиоз или внутренний эндометриоз? Основы проведения дифференциальной диагностики
}

Первый Московский Государственный медицинский университет им. И.М. Сеченова (Сеченовский университет) (Россия, Москва)

doi:10.18411/spc-15-12-2017-03

idsp: 000001:spc-15-12-2017-03

В настоящее время термином «эндометриоз» учёные называют хроническое гинекологическое заболевание, обусловленное доброкачественным разрастанием вне полости матки ткани, по морфологическим и функциональным свойствам подобной эндометрию. Клинически эндометриоз проявляется, главным образом,персистирующей болью и бесплодием.

Выделяют генитальную и экстрагенитальную формыданной патологии. Генитальный эндометриоз подразделяется на внутренний, поражающий тело матки, и наружный, мишенями которого часто становятся следующие органы: шейка матки, влагалище, промежность, ретроцервикальная область, яичники, маточные трубы, брюшина и прямокишечно-маточное углубление. 
В научных статьях последних лет их авторы всё чаще стали рассматривать внутренний генитальный эндометриоз как совершенно особое заболевание и полностью отождествлять его с термином«аденомиоз». Следовательно, врач, основываясь в клинической практике на подобных рекомендациях, будет вынужден выставлять единый диагноз. А это значит,что в обоих случаях он применит совершенно идентичную тактику лечения. Но правомерно ли это?

Поэтому цель моего исследования - это подтверждение или опровержение той точки зрения учёных, что аденомиоз возможно соотнести с внутренним эндометриозом. Задачами данной работы являются:

1) Выявление женщин групп риска по каждой патологии

2) Разработка основ дифференциальной диагностикизаболеваний, анализируя особенности их патогенеза, клинических и гистологических проявлений

Так, аденомиоз представляет собой доброкачественный патологический процесс, характеризующийся появлением в миометрии эпителиальных (железистых) и стромальных элементов эндометриального происхождения. То есть первоначально аденомиоз развивается из аномально расположенных желёз базального слоя эндометрия, а в дальнейшем затрагивает глубокие слои миометрия.Преимущественный возраст пациенток- поздний репродуктивный период (старше 42лет 3 года) и пременопауза.

Различают три степени распространения аденомиоза, причём, такназываемая первая степень аденомиоза, - это гистофизиологическая особенность матки после прерывания беременности.

Степень 1-прорастание слизистой оболочки на глубину одного поля зрения при малом увеличении светового микроскопа (2,1 мм)

Степень 2- до середины толщины стенки матки

Степень 3- в патологический процесс вовлечён весь мышечный слой

В 2013 году М.З. Р.Ф. выпустило новые клинические рекомендации, согласно которым теперь также выделяют четыре стадии аденомиоза:

стадия I - патологический процесс ограничен подслизистой оболочкой тела матки;

стадия II - патологический процесс переходит на мышечные слои;

стадия III - распространение патологического процесса на всю толщу мышечной оболочки матки до ее серозного покрова;

стадия IV - вовлечение в патологический процесс, помимо матки, париетальной брюшины малого таза и соседних органов.

Аденомиоз может быть диффузным, очаговым или узловым и кистозным. Характерное отличие от миомы матки - отсутствие капсулы и четких границ.При очаговой и узловой формах патологические изменения могут отмечаться в любых отделах матки.

Стандартным критерием, используемым в диагностике аденомиоза, является обнаружение эндометриальных желёз и стромы более, чем на глубине одного поля зрения (2,5 мм) от уровня базальной мембраны. Небольшие участки аденомиоза имеют некоторое внешнее сходство с базальными слоями эндометрия. Гистологически эти железы представляют собой неактивный или пролиферативный паттерн. Стромальный компонент превалирует над железистым, железы бедны рецепторами.

Патогенез аденомиоза остаётся неизвестным. По некоторым данным нарушается барьер между эндометрием и миометрием. Первоначально строма, а затем и железы начинают проникать в глубь миометрия по пути наименьшего сопротивления. У большинства больных развитие аденомиоза связано с пенетрацией железистого и стромального компонентов базального слоя эндометрия в миометрий, у небольшой части- с метаплазией мультипотентных мезенхимальных клеток миометрия.В очагах 
аденомиоза отсутствует секреторная трансформация, следовательно, их состояние не зависит от функционального состояния яичников и эндометрия.

Аденомиоз - заболевание с яркой клинической картиной: гипер-, -полименорея, дисменорея, увеличение матки, железодефицитная анемия (ЖДА).

Для больных аденомиозом характерно появление кровянистых выделений до и после менструации (скудные, темного «шоколадного» цвета), большая не только продолжительность менструации, но и ее обильность (при III-IV ст. распространения вплоть до меноррагий) и как следствие снижение уровня гемоглобина в крови и анемия.

В отличие от аденомиоза при эндометриозе тела матки эндометриоидные гетеротопии локализуются в области серозного покрова и прилежащем субсерозном слое миометрия. Образование эндометриоза тела матки связано с метапластическими изменениями серозного эпителия. В структурном отношении очаги эндометриоза не отличаются от таковых при аденомиозе. Очаги эндометриоза иногда могут подвергаться муцинозной метаплазии. В настоящее время подобные метапластические изменения в виде эпителия эндоцервикального типа принято именовать эндоцервикозом.

Эндометриоз чаще всего диагностируют у женщин репродуктивного возраста (25-40 лет). Средний возраст больных с эндометриоидными кистами составляет примерно 30 лет.

Существуют три клинически различные формы заболевания: эндометриоидные очаги на поверхности брюшины малого таза и яичников (перитонеальный эндометриоз), кисты яичников (эндометриомы) и солидные образования сложной структуры, включающие наряду с эндометриоидной тканью жировую и мышечнофиброзную (ректовагинальные эндометриоидные узлы); каждая из трех форм может обладать или не обладать тенденцией к инфильтративному росту.

Больные эндометриозом, как правило, имеют неблагоприятный преморбидный фон. Эндометриоз нередко сочетается с миомой матки и гиперпластическими процессами эндометрия (в 34\% случаев).

Наиболее важный клинический симптом эндометриоза - боль, связанная с менструальным циклом: тянущего характера внизу живота и в области поясницы в течение всего месяца, усиливающаяся накануне менструации и резко болезненные менструации (дисменорея). Жалобы на диспареунию (боли при половой жизни) предъявляют 26- 70\% больных генитальным эндометриозом, бесплодие (как первичное, так и вторичное) фиксируют в 46-50\% случаев. В некоторой степени болевые симптомы, могут зависеть от локализации поражений.

В ряде случаев после успешного хирургического удаления очага эндометриоза симптомы болезни самостоятельно ликвидируются и наступает беременность в естественном цикле у женщин с бесплодием.

Таблица № 1

Отличия аденомиоза от внутреннего эндометриоза

\begin{tabular}{|c|c|c|}
\hline Отличия & Аденомиоз & Внутренний эндометриоз \\
\hline Патогенез & $\begin{array}{c}\text { Пенетрация эндометрия в } \\
\text { мышечный слой }\end{array}$ & $\begin{array}{c}\text { Метаплазия мезотелия + } \\
\text { регенерация мезотелия }\end{array}$ \\
\hline $\begin{array}{c}\text { Возраст пациенток } \\
\text { (преимущественный) }\end{array}$ & $\begin{array}{c}\text { Поздний репродуктивный } \\
\text { период (старше 40 лет) } \\
\text { пременопауза }\end{array}$ & $\begin{array}{c}\text { Репродуктивный период } \\
\text { (25-40 лет) }\end{array}$ \\
\hline Клиника & Меноррагия, ЖдА & $\begin{array}{c}\text { Бесплодие, дисменорея, } \\
\text { диспареуния, тазовая боль, } \\
\text { метроррагии }\end{array}$ \\
\hline Гистологические особенности & $\begin{array}{c}\text { Стромальный компонент } \\
\text { превалирует над железистым, } \\
\text { железы бедны рецепторами }\end{array}$ & $\begin{array}{c}\text { Железы богаты рецепторами, } \\
\text { соотношение стромы и эпителия } \\
\text { вариабельно }\end{array}$ \\
\hline
\end{tabular}


Таким образом можно сделать следующие выводы:

Аденомиоз, часто употребляющийся как внутренний эндометриоз, термин ошибочный. Стоит сказать, что по данным исследований, проведённых в США, эндометриоз и аденомиоз одновременно встречаются как сопутствующие заболевания чуть менее чем у $20 \%$ женщин и требуют разных тактик ведения. Что более важно, в данной работе наглядно показано, что эндометриоз и аденомиоз- клинически различные заболевания. Их единственная общая черта-наличие эктопических желёз и стромы. В сводной таблице № 1 представлены основные критерии проведения дифференциального диагноза по патогенетическим, возрастным, клиническим и гистологическим показателям.

$$
* * *
$$

1. Стрижаков А.Н. Генитальный эндометриоз 2009

2. Эндометриоз: диагностика, лечение и реабилитация. Клинические рекомендации. М.З.Р.Ф. 2013

3. Кондриков Н.И. Патология матки, иллюстрированное руководство, 2010

4. Lobo, Gershenson, Lentz, Valea. Comprehensive Gynecology, 2014. №7

5. Vercellini P., Eskenazi B., Consonni D. et al. Oral contraceptives and risk of endometriosis: a systematic review and meta-analysis // Hum Reprod Update. - 2011. - Vol. 17. - № 2. - P. 159-170.

6. Schweppe K.-W. The Current Place of Progestins in the Treatment of Endometriosis // Expert Rev of Obstet Gynecol. - 2012. - Vol. 7. - № 2. - P. 141-148.

\section{Захарова И.В. ${ }^{1}$, Жилякова О.В. ${ }^{2}$, Белова Н.Г. ${ }^{2}$ Лечение анемии в ранние сроки беременности \\ ${ }^{1}$ ФГБОУ ВО «Сибирский государственный медицинский университет» МЗ РФ ${ }^{2}$ ФГБНУ «НИИ акушерства, гинекологии и перинатологии» Томского НИМЦ РАН} (Россия, Томск)

doi:10.18411/spc-15-12-2017-04

idsp: 000001:spc-15-12-2017-04

Одной из важных акушерских проблем является осложнение беременности анемией. Актуальность определяется высокой частотой данной патологии - 40-80 \%, а также значительным ростом акушерских и перинатальных осложнений $[1,2,3]$. В 75-90 \% случаев причиной развития анемии во время беременности является дефицит железа из-за резкого повышения потребности в этом макроэлементе во вторую половину беременности для построения тканей плода и плаценты, не обеспеченного алиментарным поступлением.

Развитие компенсаторно-приспособительных механизмов в плаценте обычно бывает усилено, особенно в ранние сроки беременности. В результате чрезмерной стимуляции компенсаторных механизмов происходит преждевременное созревание плаценты, что приводит к срыву адаптационных реакций. Плод развивается в условиях хронической гипоксии, что приводит к рождению ребёнка с выраженными признаками задержки внутриутробного развития, чаще симметричного характера $[4,5,6]$. Это определило необходимость поиска методов лечения анемии в ранние сроки беременности. Направление поиска представилось наиболее оправданным среди методов универсального неспецифического повышения компенсаторноприспособительного потенциала организма беременной, основанных на применении регуляторов энергетического обмена.

Регуляторы энергетического обмена оказывают выраженное цитопротекторное действие, препятствуют нарушению энергетического обмена в ворсинах плаценты при развитии гипоксии и ишемии. Обладая антиоксидантной и противогипоксической активностью, потенцируют действие базовой терапии направленной на улучшение кровообращения в системе мать-плацента-плод, уменьшают количество акушерских осложнений при беременности и в родах, связанных с нарушениями функции данного комплекса. Необходимость фармакологической регуляции энергетического обмена 